\title{
Profile of coordination motor abilities in elite judokas and badminton players compared to non-athletes
}

\author{
Janusz Jaworski ${ }^{1}$, Grzegorz Lech ${ }^{1}$, Tadeusz Ambroży ${ }^{1}$, Michał Żak ${ }^{2}$ \\ ${ }^{1}$ Department of Sport and Kinesiology, University of Physical Education, Kraków, Poland; ${ }^{2}$ Section of Athletics Sports \\ and Recreational Training, University of Physical Education, Kraków, Poland
}

\section{Summary}

Study aim: To determine the level and internal structure of profiles of coordination motor abilities in elite judokas and badminton players compared to a group of non-athletes.

Material and methods: The material for the study was the results collected from 12 competitors of the Polish national badminton team (age: $22.7 \pm 4.5$ ), 10 members of the national judo team who represented mean weight categories (age: $23.0 \pm 3.3$ ) and 25 non-athletes, who were male university students of the Faculty of Physical Education and Sport (age: 23.0 \pm 0.8 ). The scope of the study included basic somatic features and selected coordination motor abilities measured by means of computer tests. Results: Statistically significant differences between badminton players and judokas were obtained for reaction times (visual, auditory and selective) as well as spatial orientation. In all cases, a higher level was found for badminton players. Furthermore, analysis of differences between badminton players and non-athletes revealed statistically significant differences only for the selective reaction time. A substantial differentiation of the internal structure of the models of coordination motor abilities was obtained. This pattern was particularly noticeable in the group of judokas and badminton players, where the difference between characteristics was ca. 0.76 SD. Substantially smaller differences $(0.21 \mathrm{SD})$ between particular variables included in the coordination profile were found for non-athlete university students.

Conclusion: The lower results of the elite judokas show the need for developing the coordination motor abilities during training. The largest reserves are to be found in raising the level of reaction times and spatial orientation.

Keywords: Coordination Motor Abilities - Badminton - Judo - Elite - Non-athletes

\section{Introduction}

Badminton is one of the most popular and spectacular sports in the world. It requires cognitive abilities of a higher level in order to achieve success during competition [35]. Badminton players must perform complex movements that require adaptation to constantly changing situations on the court [29]. This leads to the necessity to make immediate decisions in conditions of time limitations. Their accuracy determines the success of the actions and the final score [8]. The analysis of matches allows for determination of the character of badminton exercise as speed-endurance. During a match, around 60 to $70 \%$ of energy is supplied from aerobic sources whereas the remaining $30 \%$ is from anaerobic sources [29]. The literature review reveals that studies have placed the most emphasis on the analyses of physiological aspects of players' level of preparation, strength and conditioning, and the level of somatic characteristics of players at various stages of training and different sports skill levels [28, 29, 32]. Another group of studies has been devoted to the biomechanical characteristics of the upper and lower limbs in badminton players [12, 16, 41]. However, the fewest studies to date have concerned the development of coordination abilities and their importance in terms of the achievement of sports results in badminton players [15].

In judo tournaments, athletes also have to compete in exceptionally variable conditions with open movement structures most frequently observed (movement stimuli come from the external environment). The effective time of a judo match for adult judokas is 4 minutes. Its temporal structure is formed by alternating bouts of fighting and breaks. If the contest is not resolved within the regular time, the match is continued based on the Golden Score rule. On the day of the competition, the medal winners 
of an elite championship usually have to compete in 4 to 6 matches. The exercise performed by judokas during a competition is usually at maximal intensity for a given level of fatigue (mainly anaerobic). This is evidenced by concentrations of lactic acid (LA) recorded in the players following the tournament matches. Its levels range from $14.6 \pm 4$ [7] to $25.1 \mathrm{mmol} / 1$ [17] in individual cases. Interestingly, aerobic capacity (reflected by minute oxygen uptake/kg body weight) also plays a significant role in this sport. It was shown that its higher values were associated with higher activity of the competitors in the second part of the fight and the Golden Score [18]. Also in badminton, many studies (overview [29]) have analysed exerciseinduced lactate levels in simulated games. However, the values obtained were significantly lower than in judokas (from $1.9 \pm 0.1$ to $12.2 \pm 2.1 \mathrm{mmol} / \mathrm{l}$ )

The material structure of the match is formed by specific actions of the competitors. A large number of these activities (the current classification of the Institute of Scientific Research - Kodokan Judo [44] includes 110 techniques) and the number of possible combinations places high demands on the competitors in terms of technical and tactical training. It is commonly believed that the competitive value of an athlete in this sport results from a combination of technical skills, the level of motor abilities and superior tactical skills [21]. Therefore, scientific research related to this sport concerns mainly the above-mentioned aspects of players' preparation $[1,2,10]$.

The analysis of the literature allows for classification of sports into separate categories differing in the structure of stimuli (internal or external), the use of energy, coordination and somatic potential, and complexity [38]. Both badminton and combat sports (such as judo) belong to the most difficult categories of sports. These sports are characterized by the highest level of variability of movement structures due to the dominance of external stimuli and open movement structures. Competitors are forced to process a lot of information in a fast-changing and unpredictable environment, which can lead to improved effectiveness of actions that require good eye-hand coordination, a high level of perception, and ability to respond adequately to situations [22]. All this also leads to the development of visual attention and making right decisions, since the effectiveness of a game or a sport fight depends on the speed of movement reactions, the level of cognitive abilities and the choice of the right movement pattern [24].

The aim of the study was to address the following research questions:

- Are there statistically significant differences in the level of selected coordination motor abilities between elite judo competitors, badminton players and nonathlete university students?

- What is the internal structure of the coordination motor ability profiles in the groups studied?

\section{Material and methods}

The research material consisted of the results collected from 12 male players of the Polish national badminton team, 10 male members of the national judo team representing medium weight categories, holding judo master ranks, and 25 non-athletes (who were male university students of the Faculty of Physical Education and Sport). All students had not practised any sport in the past or during the time of the examinations. The examinations were conducted according to the Declaration of Helsinki. Each participant gave his consent for participation in the examinations and was informed of the purpose of the study and the possibility of withdrawal from the examinations at any stage. The examinations were approved by the Bioethics Committee at the Regional Medical Chamber in Kraków, Poland (approval No. 159/KBL/OIL/2017). Examinations of judo and badminton competitors were conducted in the final phase of the competitive season in these sports.

Table 1 presents the characteristics of chronological age, training experience and basic parameters of somatic body build of study participants.

\section{Scope of the study}

The scope of the study included basic somatic characteristics: body height (b-v) and body mass, determined by a TANITA TBF-551 scale. Selected coordination motor abilities were also evaluated. For this purpose, a set of Jaworski's computer tests [33] was used to evaluate:

Table 1. Characteristics of basic somatic characteristics and training experience in the studied athletes

\begin{tabular}{lccc}
\hline \multirow{2}{*}{ Variable } & Judo $(\mathrm{n}=10)$ & Badminton $(\mathrm{n}=12)$ & Non-athletes $(\mathrm{n}=25)$ \\
\cline { 2 - 4 } & $\bar{x} \pm \mathrm{SD}$ & $\bar{x} \pm \mathrm{SD}$ & $\bar{x} \pm \mathrm{SD}$ \\
\hline Age [years] & $23.00 \pm 3.27$ & $22.69 \pm 4.49$ & $23.00 \pm 0.77$ \\
Training experience [years] & $14.20 \pm 3.39$ & $13.66 \pm 5.33$ & \\
Body height [cm] & $179.60 \pm 5.32$ & $182.03 \pm 6.09$ & $180.93 \pm 6.86$ \\
Body mass [kg] & $82.35 \pm 6.40$ & $75.60 \pm 5.51$ & $79.63 \pm 8.51$ \\
\hline
\end{tabular}


- kinaesthetic differentiation of temporal movement parameters, score in pixels,

- frequency of movements: the number of touches of squares within $15 \mathrm{~s}$ was recorded,

- mean simple reaction time to visual stimuli (10 stimuli), score in ms,

- mean simple reaction time to auditory stimuli (10 stimuli), score in ms,

- mean selective reaction time (to auditory and visual stimuli, 20 stimuli), score in ms,

- rhythmization, score in $\mathrm{ms}$,

- coupled motions, score in s (or with the number of mistakes),

- eye-hand coordination, score in s,

- spatial orientation, score in s.

All the tests were performed in a separate room that ensured silence and quiet for the study participant. The tests were always conducted from 10 a.m. to 3 p.m. in the same order. After completion of the rhythmization test, a short (10 minutes) rest was administered. Duration of the examination of each study participant was ca. 15 minutes. The tests were conducted using a Toshiba Satellite R15 portable tablet computer with a touch screen. The research protocol and variables were described in detail in the paper by Sterkowicz and Jaworski [33].

A short version of the International Physical Activity Questionnaire (IPAQ) was used to assess the level of students' general physical activity related to daily living, work, leisure time and compulsory physical activity classes [6]. Physical activity during a typical week of the academic year was also assessed.

\section{Statistical analysis}

The following statistical methods were used in the study:
1. Basic descriptive statistics of somatic characteristics and coordination motor abilities selected for the analysis were calculated.

2. The analysis of variance was based on the F test or Kruskal-Wallis $\mathrm{H}$ test, depending on the distribution and homogeneity of variance. Tukey's HSD test for unequal sample sizes and the Mann-Whitney U-test were used to examine the differences between the means from particular groups. The Bonferroni correction was used in the Mann-Whitney U-test, which consisted in dividing the level of significance $p=0.05$ by the number of comparisons [39]. The Shapiro-Wilk W test was used to examine normality of distributions. Homogeneity of variance was verified by means of Levene's test.

3. The profiles of selected coordination motor abilities in the three analysed groups were then calculated. Normalization of the results to means and standard deviations was conducted for the entire material (T scale).

Calculations were performed in the STATISTICA PL v. 12.0 package with the level of significance set at $p \leq 0.05$.

\section{Results}

Comparison of the results of the tests of coordination motor abilities between the groups studied revealed statistically significant differences in 4 cases (see Table 2).

The following homogeneous groups were formed based on multiple comparisons in the case of mean visual reaction time, mean auditory reaction time and spatial orientation:

- Badminton players and non-athlete university students,

- Judokas and non-athlete university students.

Table 2. Mean results of the tests of coordination motor abilities in groups of athletes studied

\begin{tabular}{|c|c|c|c|c|}
\hline Variable & Unit & $\begin{array}{c}\text { Judo }(\mathrm{n}=10) \\
\bar{x} \pm \mathrm{SD}(1)\end{array}$ & $\begin{array}{c}\text { Badminton }(\mathrm{n}=12) \\
\bar{x} \pm \mathrm{SD}(2)\end{array}$ & $\begin{array}{c}\text { Non-athletes }(\mathrm{n}=25) \\
\bar{x} \pm \mathrm{SD}(3)\end{array}$ \\
\hline Kinaesthetic differentiation & pixel & $39.60 \pm 21.01$ & $28.75 \pm 15.30$ & $36.00 \pm 17.14$ \\
\hline Frequency of movements & n-number & $49.70 \pm 6.53$ & $50.33 \pm 6.91$ & $48.96 \pm 9.40$ \\
\hline Mean visual reaction time & $\mathrm{ms}$ & $252.20 \pm 11.79(2 \# \#)$ & $220.83 \pm 12.56$ & $237.96 \pm 24.51$ \\
\hline Mean auditory reaction time & $\mathrm{ms}$ & $216.20 \pm 25.04(2 *)$ & $195.75 \pm 13.78$ & $209.56 \pm 15.63$ \\
\hline Mean selective reaction time & $\mathrm{ms}$ & $385.10 \pm 55.24(2 \# \#)$ & $319.91 \pm 33.53(3 \#)$ & $364.08 \pm 58.44$ \\
\hline Rhythmization & $\mathrm{ms}$ & $115.00 \pm 74.25$ & $115.42 \pm 72.54$ & $130.24 \pm 70.34$ \\
\hline Coupled motions (time) & $\mathrm{s}$ & $48.10 \pm 11.74$ & $40.58 \pm 5.66$ & $45.36 \pm 8.45$ \\
\hline Coupled motions (mistakes) & n- mistakes & $12.10 \pm 7.31$ & $13.00 \pm 5.57$ & $12.56 \pm 8.62$ \\
\hline Eye-hand coordination & $\mathrm{s}$ & $37.40 \pm 2.59$ & $34.83 \pm 3.41$ & $35.32 \pm 8.10$ \\
\hline Spatial orientation & s & $57.40 \pm 4.38(2 \# \#)$ & $48.75 \pm 4.69$ & $55.20 \pm 8.70$ \\
\hline
\end{tabular}

Stimulants are given in bold; ${ }^{*}-\mathrm{p}<0.05{ }^{* *}-\mathrm{p}<0.01$. In multiple comparisons with the Mann-Whitney U-test: \#-p $<0.016$; \#\# - p $<0.01$. 
Analysis of the means shows that the lowest value (the best result) in terms of the above indices was found in the group of badminton players while it differed significantly from the mean in the group of judokas, where it was the worst.

In terms of selective reaction time, the lowest (the best) mean occurred in the group of badminton players and it differed significantly from the mean in the groups of judokas and non-athletes forming a homogeneous group.
Based on the arithmetic means and standard deviations, normalized values of tested coordination abilities (on the $\mathrm{T}$ scale) were calculated in the entire material. The obtained results were used to determine coordination profiles in three analysed groups. The basic statistical characteristics are presented in Table 3 and illustrated graphically in Fig. 1.

A preliminary comparative analysis of the three averaged profiles of coordination motor abilities allows for

Table 3. Structure of coordination profiles in the tested groups expressed in points of the T scale

\begin{tabular}{|c|c|c|c|c|}
\hline Variable & $\bar{x} \pm \mathrm{SD}$ & Min. & Max. & $\mathrm{V}[\%]$ \\
\hline \multicolumn{5}{|c|}{ Judo $(\bar{x}=47.18)$} \\
\hline Kinaesthetic differentiation & $47.35 \pm 11.88$ & 21.12 & 62.96 & 25.08 \\
\hline Frequency of movements & $50.29 \pm 8.04$ & 38.34 & 61.74 & 15.98 \\
\hline Mean visual reaction time & $43.01 \pm 5.29$ & 32.33 & 52.97 & 12.29 \\
\hline Mean auditory reaction time & $45.32 \pm 13.38$ & 24.59 & 66.80 & 29.52 \\
\hline Mean selective reaction time & $45.09 \pm 9.76$ & 30.44 & 60.12 & 21.64 \\
\hline Rhythmization & $51.16 \pm 10.53$ & 29.62 & 61.65 & 20.58 \\
\hline Coupled motions (time) & $46.20 \pm 13.20$ & 26.07 & 63.18 & 28.57 \\
\hline Coupled motions (mistakes) & $50.63 \pm 9.70$ & 32.18 & 61.38 & 19.15 \\
\hline Eye-hand coordination & $47.19 \pm 4.14$ & 39.84 & 54.21 & 8.77 \\
\hline \multirow[t]{2}{*}{ Spatial orientation } & $45.59 \pm 5.71$ & 36.98 & 55.25 & 15.40 \\
\hline & \multicolumn{4}{|c|}{ Badminton $(\bar{x}=53.78)$} \\
\hline Kinaesthetic differentiation & $53.49 \pm 8.78$ & 32.99 & 65.22 & 16.41 \\
\hline Frequency of movements & $51.07 \pm 8.52$ & 42.04 & 69.13 & 16.68 \\
\hline Mean visual reaction time & $57.08 \pm 5.63$ & 47.14 & 65.08 & 9.74 \\
\hline Mean auditory reaction time & $56.25 \pm 7.37$ & 45.96 & 70.01 & 13.10 \\
\hline Mean selective reaction time & $56.60 \pm 5.92$ & 46.34 & 63.47 & 10.45 \\
\hline Rhythmization & $51.11 \pm 10.28$ & 32.45 & 62.22 & 20.11 \\
\hline Coupled motions (time) & $54.66 \pm 6.37$ & 44.07 & 63.18 & 11.65 \\
\hline Coupled motions (mistakes) & $49.44 \pm 7.40$ & 36.16 & 58.73 & 14.96 \\
\hline Eye-hand coordination & $51.29 \pm 5.44$ & 43.04 & 63.79 & 10.60 \\
\hline \multirow[t]{2}{*}{ Spatial orientation } & $56.88 \pm 6.12$ & 48.72 & 68.30 & 10.75 \\
\hline & \multicolumn{4}{|c|}{ Non-athletes $(\bar{x}=49.31)$} \\
\hline Kinaesthetic differentiation & $49.39 \pm 9.70$ & 21.12 & 65.22 & 19.63 \\
\hline Frequency of movements & $49.37 \pm 11.54$ & 21.10 & 67.89 & 23.37 \\
\hline Mean visual reaction time & $49.40 \pm 10.99$ & 14.39 & 64.18 & 22.24 \\
\hline Mean auditory reaction time & $48.87 \pm 8.35$ & 28.33 & 64.66 & 17.08 \\
\hline Mean selective reaction time & $48.80 \pm 10.32$ & 15.08 & 67.18 & 21.14 \\
\hline Rhythmization & $49.00 \pm 9.97$ & 24.09 & 63.78 & 20.34 \\
\hline Coupled motions (time) & $49.28 \pm 9.50$ & 27.20 & 65.43 & 19.27 \\
\hline Coupled motions (mistakes) & $50.02 \pm 11.44$ & 8.29 & 64.03 & 22.87 \\
\hline Eye-hand coordination & $50.51 \pm 12.93$ & 31.86 & 106.90 & 25.59 \\
\hline Spatial orientation & $48.46 \pm 11.31$ & 14.79 & 66.99 & 23.33 \\
\hline
\end{tabular}




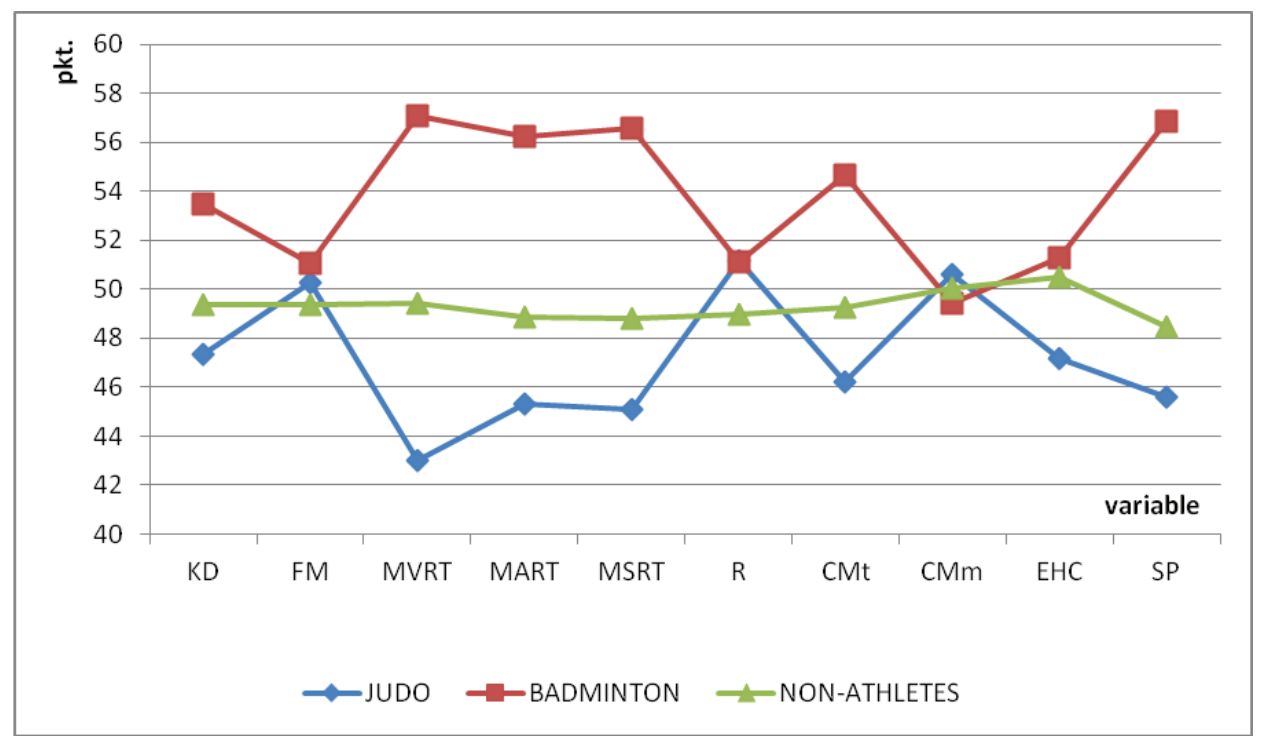

Fig. 1. Profile of coordination variables in groups of judokas, badminton players and students

KD - kinaesthetic differentiation, FM - frequency of movements, MVRT - mean visual reaction time, MART - mean auditory reaction time, MSRT - mean selective reaction time, $\mathrm{R}$ - rhythmization, CMt - coupled motions (time), CMm - coupled motions (mistakes), EHC - eye-hand coordination, $\mathrm{SP}$ - spatial orientation

capturing the characteristic system of means (Fig. 1): badminton players $>$ non-athlete university students $>$ judokas. As can be seen from Table 3, the mean score on the $\mathrm{T}$ scale in the group of judokas for all ten variables was 47.18 points. On the other hand, differentiation between characteristics was around 0.76 of the standard deviation. It is also important to emphasize the substantial differentiation in individual abilities included in the profile. In general, coefficients of variation for particular abilities ranged from $13 \%$ to $30 \%$. The only exception to this rule was the coefficient of variation for visual-motor coordination (about 9\%). In the group of badminton players, the mean of all variables was as high as 53.78 points, with the range of differentiation between characteristics of 0.76 of the standard deviation. It should also be emphasized that the differences within the analysed abilities were much smaller than in the group of judokas. In general, coefficients of variation ranged from $10 \%$ to $17 \%$. The only exception to this rule was the coefficient of variation for rhythmization (ca. 20\%). In the group of non-athletes, the mean for the entire analysed coordination profile was 49.31 points. The differentiation between characteristics was 0.21 of the standard deviation. Most coefficients of variation in individual coordination motor abilities were around $20 \%$. Very interesting information can be obtained from the analysis of differentiation within the three groups by taking into account individual coordination motor abilities. As can be seen from Fig. 1, there is virtually no differentiation for: coupled motions (mistakes), frequency of movements and rhythmization. Definitely, the greatest differences were observed between judokas and badminton players for visual reaction time, which was 14.07 points. Furthermore, higher results (by about 11 points) were found in badminton players for complex reaction time, spatial orientation and auditory reaction time. The smallest differences in favour of badminton players were obtained for kinaesthetic differentiation (6.14 points) and coupled motions - time (8.46 points).

Table 4 presents the level of general physical activity of students related to activities of daily living, work and leisure time, and obligatory physical activity classes included in the university curriculum.

Analysis of the results obtained based on the IPAQ questionnaire revealed that the average level of overall

Table 4. Level of physical activity of university non-athlete students (MET-minutes/week)

\begin{tabular}{cccccc}
\hline Physical activity & Vigorous intensity & Moderate intensity & Walking & Obligatory activity & Total \\
\hline $\bar{x}$ & 1937.27 & 730.91 & 1278.75 & 663.75 & 4610.68 \\
SD & 956.47 & 548.93 & 1016.00 & 117.99 & 1487.61 \\
$\mathrm{X}_{\min }$ & 320.00 & 0.00 & 0.00 & 495.00 & 2229.00 \\
$\mathrm{X}_{\max }$ & 3840.00 & 2160.00 & 3564.00 & 742.50 & 7474.50 \\
\hline
\end{tabular}


physical activity of non-athlete students during the week was $4610.68 \mathrm{MET}-\mathrm{min} /$ week.

\section{Discussion}

Most sports require high-performance processing of neurophysiological information from athletes. Coordination motor abilities are therefore among the important factors in the achievement of a champion sports skill level. With a very similar level of fitness-related abilities, reserves in improving results are expected to lie in improving coordination and perceptual abilities [25]. Hence, the search for their importance to many different sports is obvious, including in judo and badminton [11, 14, 19, 20, 23, 30, 34].

Badminton is one of the fastest racket sports, with the maximum shuttlecock flight speed reaching almost 500 $\mathrm{km} / \mathrm{h}$ [29]. For this reason, players must have a high level of divisibility of attention, precision and accuracy of movements, speed of movements and spatial orientation and anticipation [31]. Among the identified coordination motor abilities, the most frequently analysed were reaction time levels of badminton players relative to comparative groups, mostly non-athlete peers or competitors from other sports $[3,5,9,34]$. The literature review shows that badminton players have better reaction times compared to non-athletes from control groups. These findings are consistent with our results obtained for all three analysed reaction time types. The analysis of the system of normalized differences clearly indicates that the largest differences are observed between the group of badminton players and judokas. These differences were also statistically significant in four cases. This may indicate the importance of the reaction time in badminton, and regular training of this ability. Determining the level of these characteristics among young athletes can be helpful for the effectiveness of the recruitment and selection process for badminton [3].

The reaction time depends on the type of stimulus to which the respondents react. The shortest reaction times were found for auditory stimuli, followed by visual stimuli, whereas the longest were found for selective reaction [42]. The results of our study confirmed this characteristic distribution of arithmetic means of reaction times in all three analysed groups.

Badminton belongs to the group of sports with nonstandard properties and requires a high level of movement coordination. Several comparative studies have confirmed the importance of visual and motor coordination and reaction time for the effectiveness of the game $[3,15,37,40]$. The role and importance of coordination motor abilities in the teaching of game technique has also been emphasized $[27,32,36]$.
Jaworski and Żak drew attention to the clear effect of coordination variables in the development of the sports skill level at three stages of badminton training [15]. These authors postulated the early development of coordination motor abilities with a higher degree of organisation and specificity: spatial orientation, selective reaction time, coupled motions and kinaesthetic differentiation.

The worst values of mean reaction times (visual, auditory and selective) and spatial orientation recorded in judo players should be explained by the specific training in this sport. It is likely that the development of strength and endurance limits the development of these aspects of coordination motor abilities. Previous studies have demonstrated that the above-mentioned indices correlated with the indices of courses of the match in the group of adult athletes [18]. Spatial orientation was correlated with the activity of the athletes, visual reaction time was linked to activity in the first part of the match, auditory reaction time with activity in the second part of the match, and selective reaction time with the effectiveness of actions in the first part of the fight. The fact that the values of these indices were higher than in the population and that their values were related to the course of the match clearly indicates the need for developing them.

The analysis of the results obtained in our study indicates a relatively high level of coordination motor abilities among non-athletes. The reasons for this status can be found in the high physical activity of physical education and sport students, which probably results from their interests and the following the obligatory university curriculum. According to the generally accepted recommendations [6], all students showed high levels of physical activity. Previous studies have shown that according to the adopted classification, physical activity of the university students of the University School of Physical Education in Kraków is high [43]. The mean level of overall physical activity of the examined Polish men was 5.953 METminutes/week [4]. It is generally accepted that regular physical activity has a positive effect on the level of both fitness and coordination abilities. Thus, the high level of coordination motor abilities in the group of university students may have been related to their high physical activity. It is known that coordination motor abilities show a varied strength of genetic determinants. Depending on the type, they can be trained to a significant degree $[13,26]$.

\section{Conclusions}

In the case of most of the analysed abilities, the best values were found in the group of badminton players, worse in non-athlete university students, and the worst in judokas. Significant differences concerned reaction times (visual, auditory and selective) and spatial orientation. 
The analysis of averaged profiles of coordination motor ability allows a characteristic system of mean values to be found: badminton players $>$ untrained university students $>$ judokas. It should also be emphasized that the internal profiles in the group of judokas and badminton players were relatively diversified. The lower results in judokas indicate reserves in coordination preparation, especially in terms of reaction times and spatial orientation.

Despite the domination of open motor structures in both sports (motor stimuli come from the external environment), it is likely that the model of competition, i.e. direct contact, and the related transfer of significant muscle torques, affect the lower values of the tested coordination abilities in judo players. The observed differences should be interpreted with caution since the measurement concerned general rather than special motor coordination (manifested in sports fighting conditions).

\section{Conflict of interest: Authors state no conflict of interest.}

\section{References}

1. Adam M., Klimowicz P., Pujszo R. (2016) Judoists' tactical and technical efficiency during the World Championships in 2014 and 2015. Balt. J. Health Phys. Act., 8(2): 19-28.

2. Adam M., Wolska B., Tabakov S. (2018) Ways of settling a judo fight at consecutive stages of sports competitions. Balt. J. Health Phys. Act., 10(1): 30-37. DOI: 10.29359/ BJHPA.10.1.03.

3. Bańkosz Z., Nawara H., Ociepa M. (2013) Assessment of simple reaction time in badminton players. Trends Sports Sciences, 1(20): 54-61.

4. Bednarek J., Pomykała S., Bigosińska M., Szyguła Z. (2016) Physical activity of Polish and Turkish university students as assessed by IPAQ. Cent. Eur. J. Sport Sci. Med., 4(16): 13-22. DOI: 10.18276/cej.2016.4-02.

5. Bhabhor M., Vidja K., Dubal G., Padalia M., Joshi V. (2013) A comparative study of visual reaction time in badminton players and healthy controls. Indian J. Physiol. Pharmacol., 57(4): 439-442.

6. Biernat E., Stupnicki R., Gajewski A.K. (2007) International Physical Activity Questionnaire IPAQ: Polish version (Międzynarodowy Kwestionariusz Aktywności Fizycznej (IPAQ) - wersja polska). Wychowanie Fizyczne i Sport, 51(1): 47-54. [in Polish].

7. Bonitch-Domínguez J., Bonitch-Góngora J., Padial P., Feriche B. (2010) Changes in peak leg power induced by successive judo bouts and their relationship to lactate production. J. Sports Sci., 28(14): 1527-1534. DOI: 10.1080/02640414.2010.512641.

8. Chansrisukot G., Suwanthada S., Intiraporn C. (2015) Cognitive psychological training in combination with explosive power training can significantly enhance responsiveness of badminton players. J. Exerc. Physiol., Online. 18(4): 17-32.

9. Dube S.P., Mungal S.U., Kulkarni M.B. (2015) Simple visual reaction time in badminton players: A comparative study. Natl. J. Physiol. Pharm. Pharmacol., 5(1): 18-20. DOI: 10.5455/njppp.2015.5.080720141.

10. Franchini E., Del Vecchio F.B., Matsushigue K.A., Artioli G.G. (2011) Physiological profiles of elite judo athletes. Sports Med., 41(2): 147-166. DOI: 10.2165/11538580000000000-00000.

11. Hijazi M. (2013) Attention, visual perception and their relationship to sport performance in fencing. J. Hum. Kinet., 39: 195-201. DOI: 10.2478/hukin-2013-0082.

12. Hong Y., Wang S.Y., Lam W.K., Cheung, J.T. (2014) Kinetics of badminton lunges in four directions. J. Appl. Biomech., 30(1): 113-118. DOI: 10.1123/jab.2012-0151.

13. Jaworski J., Klimek A.T., Wieczorek T. (2010) Family studies of somatic and functional characteristics in the polish rural population. Biol. Sport, 27(3): 207-212.

14. Jaworski J., Lech G., Żak M., Madejski E., Szczepanik K. (2017) The level of selected coordination abilities in badminton players at various ages and sport skill levels as compared to non-athletes. Balt. J. Health Phys. Act., 9(3): 33-43.

15. Jaworski J., Żak M. (2015) The structure of morphofunctional conditions determining the level of sports performance of young badminton players. J. Hum. Kinet., 47: 129-137. DOI: 10.1515/hukin-2015-0077.

16. Kuntze G., Mansfield N., Sellers W. (2010) A biomechanical analysis of common lungs tasks in badminton. J. Sports Sci., 28(2): 183-191. DOI: 10.1080/02640410903428533.

17. Laskowski R., Kujach S., Smaruj M., Grzywacz T., Łuszczyk M., Marek A., Ziemann E. (2012) Lactate concentration during one-day male judo competition: A case study. Arch. Budo., 8(1): 51-57. DOI: 10.12659/AOB.882651.

18. Lech G., Jaworski J., Krawczyk R. (2007) Relationship between coordination motor abilities and the course of judo fights and level of achievement in adult judo athletes (Poziom koordynacyjnych zdolności motorycznych a przebieg walk i poziom osiagnięć seniorów judo). Antropomotoryka, 17(40): 63-69. [in Polish].

19. Lech G., Jaworski J., Lyakh V., Krawczyk R. (2011) Effect of the level of coordinated motor abilities on performance in junior judoka. J. Hum. Kinet., 30: 153-160. DOI: $10.2478 / \mathrm{v} 10078-011-0083-0$.

20. Lech G., Sertić H., Sterkowicz S., SterkowiczPrzybycień K., Jaworski J., Krawczyk R. (2014) Effects of different aspects of coordination on the fighting methods and sport skill level in cadet judo contestants. Kinesiology, 46(1): 69-78.

21. Lech G., Sterkowicz S. (2004) The commencement age of training and its effects on technical preferences and 
achievements attained by judo contestants. Hum. Mov., 5(1): 42-47.

22. Lees A. (2003) Science and the major racket sports: A review. J. Sports Sci., 21(9): 707-732. DOI: 10.1080/0264041031000140275.

23. Lesiakowski P., Krzepota J., Zwierko T. (2017) The Differentiation of visual sensorimotor processes in the representatives of various sport disciplines. Cent. Eur. J. Sport Sci. Med., 19(3): 43-53. DOI: 10.18276/cej.2017.3-04.

24. Liao K.F., Meng F.W., Chen Y.L. (2017) The relationship between action inhibition and athletic performance in elite badminton players and non-athletes. J. Hum. Sport Exerc., 12(3): 574-581. DOI: 10.14198/jhse.2017.123.02.

25. Lum J., Enns J.T., Pratt J. (2002) Visual orienting in the college athletes: Explorations of athlete type and gender. Res. Q. Exerc. Sport., 73: 156-167. DOI: 10.1080/02701367.2002.10609004.

26. Lyakh V., Jaworski J., Wieczorek T. (2007) Genetic endowment of coordination motor abilities a review of family and twin research. J. Hum. Kinet., 17: 25-40.

27. Mooney R., Mutrie N. (2000) The effects of goal specificity and goal difficulty on the performance of badminton skills in children. Pediatr. Exerc. Sci., 12(3): 270-283.

28. Ooi C.H., Tan A., Ahmad A., Kwong K.W., Sompong R., Ghazali K.A., Liew S.L., Chai W.J., Thompson M.W. (2009) Physiological characteristics of elite and sub-elite badminton players. J. Sport Sci., 27(14): 1591-1599. DOI: $10.1080 / 02640410903352907$.

29. Phomsoupha M., Laffaye G. (2015) The science of badminton: Game characteristics, anthropometry, physiology, visual fitness and biomechanics. Sports Med., 45(4): 473-495. DOI: 10.1007/s40279-014 - 0287-2.

30. Pion J., Segers V., Fransen J., Debuyck G., Deprez D., Haerens L., Vaeyens R., Philippaerts R., Lenoir M. (2015) Generic anthropometric and performance characteristics among elite adolescent boys in nine different sports. Eur. J. Sport Sci., 15(5): 357-366. DOI: 10.1080/17461391.2014.944875.

31. Poliszczuk T., Mosakowska M. (2009) Interreactions of peripheral perception and ability of time-movement anticipation in high class competitive badminton players. Studies in Physical Culture and Tourism, 16(3): 259-265.

32. Sakurai S., Ohtsuki T. (2000) Muscle activity and accuracy of performance of the smash stroke in badminton with reference to skill and practice. J. Sport Sci., 18(11): 901-914. DOI: 10.1080/026404100750017832.

33. Sterkowicz S., Jaworski J. (2013) Participation in computer games vs. Coordination motor abilities and body composition in boys from rural areas of Poland. Hum. Mov., 14(1): 4-10. DOI: 10.2478/v10038-012-0048-8.

34. Subramanyam V., Manilal K.P. (2014) The interrelationship of simple visual reaction time, choice visual reaction time and attention control among the national level players of selected disciplines of sports authority of India. Int. J. Sports Sci. Fitness, 4(2): 157-164.

35. van de Water T., Huijgen B., Faber I., Elferink-Gemser M. (2017) Assessing cognitive performance in badminton players: A reproducibility and validity study. J. Hum. Kinet., 55(1): 149-59. DOI: 10.1515/hukin-2017-0014.

36. Wang S., Zhang J., Yin X. (2009) A research on performance of perceptual-motor skill training for badminton. Journal of Beijing Sport University, 32(9): 46.

37. Wang S., Yan C., Zhang J. (2008) A research on evaluation target reaction ability correctly for badminton players in the process of appraising perceptual-motor skill. Journal of Beijing Sport University, 31(6): 779-781.

38. Wang C.H., Chang C.C., Liang Y.M., Shih C.M., Chiu W.S., Tseng P., Hung D.L., Tzeng O.J., Muggleton N.G., Juan C.H. (2013) Open vs. closed skill sports and the modulation of inhibitory control. PLOS ONE, 8(2):e55773. DOI: 10.1371/journal.pone.0055773.

39. Wilcox R. (1987) New statistical procedures for the social sciences. Modern solutions to basic problem. Hillsdale NJ: Lawrence Erlbaum Associates.

40. Yuan Y.W.Y., Fan X., Chin M., So R.C.H. (1995) Handeye co-ordination and visual reaction time in elite badminton players and gymnasts. New Zel. J. Sports Med., 23(3): 19-22.

41. Zhao X., Li S. (2019) A biomechanical analysis of lower limb movement on the backcourt forehand clear stroke among badminton players of different levels. Appl. Bionics. Biomech., Article ID 7048345. DOI: $10.1155 / 2019 / 7048345$.

\section{Internet sources}

42. http://www.cognaction.org/cogs105/readings/clemson. rt.pdf - Accessed January 16, 2019

43. https://sites.google.com/site/theipaq/scoring-protocol Accessed January 24, 2019

44. http://kodokanjudoinstitute.org/en/waza/list/ - Accessed January 24, 2019

\section{Received 11.05.2019 \\ Accepted 08.01.2020}

(C) University of Physical Education, Warsaw, Poland 